\title{
Effects of Acute Exercise on Long-Term Memory
}

By: Jeffrey D. Labban and Jennifer L. Etnier

Labban, J.D., \& Etnier, J.L. (2011). Effects of acute exercise on long-term memory. Research Quarterly for Exercise and Sport, 82(4), 712-721.

This is an Accepted Manuscript of an article published by Taylor \& Francis in Research Quarterly for Exercise and Sport in 2011, available online:

http://wwww.tandfonline.com/10.1080/02701367.2011.10599808

****() Taylor \& Francis. Reprinted with permission. No further reproduction is authorized without written permission from Taylor \& Francis. This version of the document is not the version of record. Figures and/or pictures may be missing from this format of the document. $* * *$

\section{Abstract:}

In this study, we tested the effect of acute exercise on long-term memory, specifically the timing of exercise relative to the memory challenge. We assessed memory via paragraph recall, in which participants listened to two paragraphs (exposure) and recounted them following a 35-min delay. Participants $(n=48)$ were randomly assigned to one of three groups: exercise prior to exposure, exercise after exposure, or no-exercise. Exercise consisted of $30 \mathrm{~min}$ on a cycle ergometer, including $20 \mathrm{~min}$ at moderate intensity. Only the exercise-prior group recalled significantly more than the control group $(\mathrm{p}<.05)$. Differences among the exercise groups failed to reach significance $(\mathrm{p}=.09)$. Results indicated that acute exercise positively influenced recall and that exercise timing relative to memory task may have an impact on this effect.

Keywords: Aerobic | Ergometer | Physical Activity | Recall

\section{Article:}

The scientific and medical communities generally agree that exercise is beneficial for body and mind. Although much of the literature examining the relationship between exercise and cognitive performance has traditionally focused on chronic exercise paradigms, a growing body of research has focused on the effects of acute exercise (Audiffren, Tomporowski, \& Zagrodnik, 2009; Brisswalter, Collardeau, \& René, 2002; Davranche \& McMorris, 2009; Lambourne, Audiffren, \& Tomporowski, 2010; Pontifex, Hillman, Fernhall, Thompson, \& Valentini, 2009; Tomporowski, 2003). The cognitive tasks most often examined in the acute exercise literature include reaction time (Arcelin \& Brisswalter, 1999; Arcelin, Brisswalter, \& Delignieres, 1997; Arcelin, Delignieres, \& Brisswalter, 1998; Brisswalter, Arcelin, Audiffren, \& Delignieres, 1997; Brisswalter, Durand, Delignieres, \& Legros, 1995; Collardeau, Brisswalter, \& Audiffren, 2001; Davranche, Burle, Audiffren, \& Hasbroucq, 2006; Kamijo, Nishihira, Higashiura, \& Kuroiwa, 2007; Scott, McNaughton, \& Polman, 2006), information processing (Aks, 1998; Davranche, Burle, Audiffren, \& Hasbroucq, 2005; Hogervorst, Riedel, Jeukendrup, \& Jolles, 1996; Hogervorst, Riedel, Kovacs, Brouns, \& Jolles, 1999; Kamijo et al., 2004; Travlos \& Marisi, 1995; 
Welsh, Davis, Burke, \& Williams, 2002), and elements of executive functioning (Emery, Honn, Frid, Lebowitz, \& Diaz, 2001; Hillman, Snook, \& Jerome, 2003; Kubesch et al., 2003; McMorris \& Graydon, 1996; McMorris et al., 1999; Netz, Tomer, Axelrad, Argov, \& Inbar, 2007).

When they reviewed this literature meta-analytically, Etnier et al. (1997) concluded there was a small but significant positive effect of acute exercise on cognitive performance $(\mathrm{ES}=0.16$, $\mathrm{SD}=0.6$ ). In two narrative reviews of the acute exercise literature, Brisswalter and colleagues (2002) and Tomporowski (2003) both concluded that acute exercise has a positive effect on cognitive performance. In both reviews, the authors pointed out that exercise intensity and duration are important considerations in testing the effect on cognition. Tomporowski (2003) reported that results were more consistent when participants performed exercise protocols at submaximal intensities for durations of 20-60 min. Brisswalter and colleagues (2002) echoed this sentiment, arguing that exercise is more likely facilitative when submaximal protocols are sufficient to elicit physiological changes (e.g., elevations in blood catecholamine levels) and last between 20 and $60 \mathrm{~min}$. Authors in both reviews posited that short-to-moderate duration, highintensity exercise protocols performed by more fit individuals could also result in cognitive function gains. In all cases, it was agreed that longer protocols leading to dehydration and fatigue were likely to decrease performance on cognitive tasks.

Although there is evidence for facilitative effects of acute exercise on cognitive performance in general, the effects on long-term memory are less clear and have been examined to a lesser extent. Even the aforementioned narrative reviews conflict as to whether the positive effect of acute exercise extends to long-term memory. Based on the findings of four studies, Tomporowski (2003) concluded that acute exercise had no effect on long-term memory. However, it is important to note that all study designs in this review incorporated at least one factor suggested to have deleterious effects on cognitive performance. These include encoding of to-be-remembered material during exercise (Sjoberg, 1980), high-duration exercise protocols leading to dehydration and fatigue (Cian, Barraud, Melin, \& Raphel, 2001; Cian et al., 2000), or intense exercise to exhaustion (Tomporowski, Ellis, \& Stephens, 1987). This explanation is consistent with the conclusion drawn by Brisswalter and colleagues (2002) that performance on long-term memory tasks might be improved by acute exercise paired with fluid ingestion to prevent the deleterious effects of dehydration.

In empirical research conducted since these narrative reviews, positive effects for acute exercise on several types of memory were reported. Tomporowski and colleagues (2005) found that acute bouts of aerobic exercise performed by highly trained individuals could be linked to positive gains in serial addition tasks, which include, among other things, components of working memory. Other empirical research also revealed benefits of acute aerobic exercise on decision making and reaction time tasks predicated on working memory capacity (Pontifex et al., 2009; Sibley \& Beilock, 2007). In contrast, Winter et al. (2007) tested the ability to recall a novel vocabulary using associative learning methods and reported no differences between exercise groups and a control group in immediate recall or recall after 1 week or 8 months. The exercise conditions were a 40-min low-impact running condition (median heart rate $=140 \mathrm{bpm}$ ) and an "intense" condition consisting of two 3-min sprints separated by a 2-min break. In recent research examining long-term memory in particular, Potter and Keeling (2005) found that 10 min of lightto-moderate aerobic exercise produced beneficial effects on a free-recall task incorporating elements of working and long-term memory. Coles and Tomporowski (2008) found that 40 min of moderate exercise on a cycle ergometer produced improvements in certain portions of a freerecall, list-learning task. Finally, a study by Pesce, Crova, Cereatti, Casella, and Belluci (2009) 
tested children's immediate and delayed recall of a word list at baseline (not following any type of exercise) and after an acute bout of physical activity (circuit training and team games). They observed better immediate recall after team games as compared to baseline testing. Results also demonstrated better delayed recall following participation in either team games or circuit training as compared to baseline.

Given the mixed findings of the previous literature, the primary purpose of the current study was to test the effects of acute aerobic exercise on long-term memory. In examining the methodology of studies that have and have not shown a positive effect of acute exercise on memory, it is apparent that those with null results are characterized by elements (e.g., dehydration or exhausting intensity) that might have limited the ability to clearly demonstrate a relationship between acute exercise and cognition. Therefore, we used a study design that introduced neither dehydration nor exhaustion. In particular, the design consisted of a moderate exercise duration performed at a moderate and individualized intensity. Additionally, we used a standardized paragraph recall test to assess long-term memory, and encoding did not occur during the exercise bout. We hypothesized that participants engaging in acute exercise would perform better on the recall task than those not performing any exercise.

An additional consideration in such research is the length of the delay period prior to asking participants to recall the information. Evidence suggests that assessment timing is an important factor, specifically when testing long-term memory. Revelle and Loftus (1992) summarized literature indicating that higher activation levels temporally proximal to encoding may inhibit short-term recall but facilitate recall after delays of more than $30 \mathrm{~min}$. This suggests that moderate-to-intense exercise should not necessarily be expected to improve information recall assessed shortly after an exercise protocol, when arousal is still well above baseline. This is consistent with recent research (Coles \& Tomporowski, 2008) showing that exercising prior to list learning did not facilitate immediate recall, but recall following a 12-min delay showed an advantage for the exercise group over the control group for primacy and recency portions of the lists. Thus, in this study, we assessed recall 35 min following exposure to the paragraph.

An additional purpose of this study was to extend the literature by examining the influence of exposure timing to the to-be-remembered information relative to the exercise bout. Because long-term memory requires a transference process that takes place between the time of information exposure and the time of recall, it is conceivable the timing of exercise relative to this process would have an impact on any effects exercise might have on that process. This is especially likely given the proposed underlying mechanisms for the effects of acute exercise on cognitive performance. In particular, it has been proposed that changes in catecholamines, brain derived neurotrophic factor (BDNF), and serotonin are responsible for the cognitive benefits of acute exercise (Ahmadiasl, Alaei, \& Hänninen, 2003; McGaugh, 1989; Meeusen et al., 1996; Soya et al., 2007). This was supported by the findings of Winter et al. (2007) who reported that immediate and long-term learning correlated with sustained, exercise-induced increases in BDNF levels as well as larger absolute levels of dopamine and epinephrine. By using an activity of moderate intensity and duration in the current study, we provided an opportunity for some of these physiological processes to occur. If increases in neurochemicals are the mechanism by which exercise affects memory, these results would seem to suggest activation prior to encoding would be the most likely scenario for observing a facilitative effect. Thus, a second goal of this study was to assess the impact of exercise timing on long-term memory relative to exposure to the memory task. We hypothesized that the group engaging in aerobic exercise prior to the 
presentation of novel information would recall the paragraphs better than both the no-exercise group and the group exercising immediately after paragraph presentation.

\section{Method}

\section{Participants}

The university's institutional review board reviewed and approved all protocol and procedures pertaining to this study. Forty-eight young adults (15 men, 33 women; M age = 22.02 years) were recruited from the University of North Carolina-Greensboro campus. The ethnic breakdown of the sample was representative of Guilford County: 33 Caucasians, 10 African Americans, 3 Asians, 1 Hispanic, and 1 participant self-identifying as "other." All provided informed consent to participate in the study, were apparently healthy, and completed a questionnaire adapted from the Physical Activity Readiness Questionnaire (PAR-Q) to ensure they could safely complete the submaximal exercise protocol. Qualifying participants were randomly assigned to one of three groups: exercise-prior group, exercise-after group, or control group. Groups were matched by gender prior to group assignment to ensure the breakdown was equivalent across groups ( 5 men, 11 women). The literature has been clear that women consistently exhibit an advantage in verbal recall over men (Herlitz, Nilsson, \& Backman, 1997; Herlitz \& Yonker, 2002; Kimura \& Seal, 2003; Kramer, Delis, \& Daniel, 1988; Maitland, Herlitz, Nyberg, Backman, \& Nilsson, 2004; Ragland, Coleman, Gur, Glahn, \& Gur, 2000); thus, it was critical that gender distribution across groups was comparable.

\section{Materials}

Long-Term Memory. To assess long-term memory (recall) performance, we used the standard New York University Paragraphs for immediate and delayed recall, a subtest of the Guild Memory Test (Crook, Gilbert, \& Ferris, 1980; Gilbert \& Levee, 1971; Gilbert, Levee, \& Catalano, 1968). This test is similar in construct, execution, and scoring to the Wechsler Logical Memory test (Wechsler, 1997). It is a widely used and accepted assessment tool for memory, with good split-half reliability $\left(\mathrm{r}^{2}=.87\right.$; Gilbert, Levee, \& Catalano, 1973). The test involves reading two brief paragraphs (Story A and Story B) to participants and asking them to recall each one immediately after it is read. The reading and immediate recounting sequence is performed once for Story A, and twice for Story B. The second reading and recounting of Story B is performed to ensure that enough material is learned at exposure to adequately assess delayed recall. Then, after a delay, participants are asked to recall each story again, as close to verbatim as possible. Each paragraph is composed of two sentences and is four lines in length. Paragraphs are divided into units (one to three words), which are scored as correctly recalled when recounted verbatim. Story A is composed of 21 units, and Story B is composed of 22 units. Following a 35-min delay, units correctly recounted from Stories A and B are summed to obtain a single score for delayed recall. A single coder scored the test; however, to reduce the potential for scoring ambiguity only verbatim responses were coded as correct.

Physical Activity Level. We used the National Health Interview Survey, Part E (NHIS; Benson \& Marano, 1998) to assess current lifestyle physical activity levels. The NHIS has been found to exhibit high validity when used among a target population ages 18-34 years (Weiss et al., 1990). Participants reported their various physical activity levels for the previous 2 weeks. 
Average metabolic equivalent values for each reported activity were taken from the Compendium of Physical Activities Tracking Guide (Ainsworth, 2002). We then calculated values to assess daily energy expenditure via physical activity ( $\mathrm{kcal} / \mathrm{kg} /$ day). Although comprehensive in types of leisure time physical activities covered, the questionnaire is brief and was completed in less than 10 min. Participants also completed a brief demographic questionnaire.

Ratings of Perceived Exertion. Because it has been suggested that detrimental effects from fatigue could mask the beneficial effects of exercise on performance (Tomporowski, 2003; Tomporowski \& Ellis, 1986), it was important that each individual exercised at what he or she considered a moderate intensity to ensure the exercise was nonfatiguing. Thus, participants exercised at a self-determined moderate intensity level using the Borg scale of perceived exertion (ratings of perceived exertion; RPE) with ratings of 13-15 as their parameter. This scale has been shown to be psychometrically sound, with reliability coefficients above .90 and validity coefficients at .77 and higher (Borg, 1998). Borg's scale has also been shown to be a valid tool for regulating exercise intensity on a cycle ergometer (Dunbar et al., 1992). RPE measures were taken at the beginning of and every 5 min during exercise and rest phases.

Heart Rate. Heart rate (HR) was used as an additional assessment of physiological arousal and exertion and was monitored via a short-range radio telemetry device, the Polar F6 Heart Rate Monitor, (Kempele, Finland). The monitors included an elastic band, which held a padded HR sensor and transmitter below the participant's sternum. Also included was a wristband receiver/monitor, which continuously recorded and displayed HR. Resting HR was obtained from all participants at the beginning of the appointment.

Acute Exercise Protocol. The protocol was a single bout of aerobic exercise of moderate intensity and duration on a cycle ergometer. The protocol began with a 5-min warm-up, during which resistance was set at $0.5 \mathrm{kp}$. Following the warm-up, resistance was increased to $1 \mathrm{kp}$, and participants were asked to pedal at a rate resulting in RPE ratings within the prescribed 13-15 range. Participants exercised at a moderate intensity for $30 \mathrm{~min}$ to control for factors associated with exercise bouts of high intensity and longer duration (e.g., fatigue, dehydration) that might also influence memory performance. For this reason also, water was provided to the participants throughout the session. Exercise concluded with a 5-min cool-down period.

\section{Procedure}

Each person in the exercise-prior group began by completing the exercise protocol, immediately after which the paragraphs were read to the participant (paragraph exposure). The investigator read Story A and then had the participant immediately recount the story as close to

\begin{tabular}{|c|c|c|c|}
\hline \multicolumn{4}{|c|}{ Exercise-Prior } \\
\hline Exercise & Exposure & Rest & Recall \\
\hline (30 Minutes) & \multicolumn{3}{|c|}{ (30 Minutes) } \\
\hline \multicolumn{4}{|c|}{ Exercise-After } \\
\hline Rest & Exposure & Exercise & Recall \\
\hline (30 Minutes) & \multicolumn{3}{|c|}{ (30 Minutes) } \\
\hline \multicolumn{4}{|c|}{ Control } \\
\hline Rest & Exposure & Rest & Recall \\
\hline
\end{tabular}


verbatim as possible. The investigator then read Story B and then asked the participant to recount the story as close to verbatim as possible. Story B was read and recounted a second time. To reduce rehearsal and expectation of later story recall, participants completed the Stroop Task as a distracter at the conclusion of this phase. Participants then rested quietly for $30 \mathrm{~min}$. Their HR and RPE were monitored every 5 min during exercise and rest. The exercise-prior group completed the delayed recall testing immediately following the rest phase. The protocol for the exercise-after group differed from the exercise-prior group only in that they rested quietly prior to paragraph exposure and completed the exercise immediately following paragraph exposure. They then completed the delayed recall testing. Participants in the control group did not exercise at all but rested quietly before and after paragraph exposure. This design is illustrated in Figure 1 . At the session end, we asked all participants if they expected to be required to recall the stories and if they spent time rehearsing the stories after hearing and recounting them.

\section{Analysis}

HR and RPE were assessed at 5-min intervals during rest and exercise sessions. We used a mixed analysis of variance (ANOVA) with repeated measures for time to ensure there were no between-groups differences in RPE or HR during exercise. In comparing RPE and HR during the exercise conditions, the between-groups factor consisted of two levels (exercise-prior group prior to exposure, exercise-after group after exposure). Greenhouse-Geisser adjustment was used when sphericity could not be assumed. We also used a one-way ANOVA to determine if long-term recall performance was significantly different between groups. To follow up on any significant $\mathrm{F}$ values, we used Tukey's honestly significant differences (HSD) post hoc tests. Finally, partial etasquared $\left(\eta p^{2}\right)$ is presented as a measure of the omnibus effect size for significant ANOVAs, and Cohen's $d$ was calculated as a measure of the effect size comparisons between each possible group pairing.

\section{Results}

\section{Lifestyle Activity}

A one-way ANOVA comparing average energy expenditure per day revealed no significant between-groups differences, $\mathrm{F}(2,45)=0.108, \mathrm{p}>.05$.

\section{HR and RPE}

Figure 2 shows HR at 5-min intervals for each group, and Figure 3 shows RPE values for each group. In comparing the responses of exercise-prior and exercise-after groups to the exercise protocol, a repeated-measures mixed ANOVA revealed the expected significant effect for time for both HR, $\mathrm{F}(2.58,77.31)=212.19, \mathrm{p}<.001$, and RPE, $\mathrm{F}(3.57,107.08)=125.17, \mathrm{p}<.001$. However, neither the main effect for group nor the Group $\mathrm{x}$ Time interaction was significant for either HR, $\mathrm{F}(2.58,77.31)=1.60, \mathrm{p}>.05$, or RPE, $\mathrm{F}(3.57,107.08)=0.273, \mathrm{p}>.05$. The average $\mathrm{HR}$ across the moderate intensity portion of the exercise sessions was $146.53 \mathrm{bpm}(\mathrm{SD}=21.6)$, which represents approximately $74 \%$ of age-predicted (using the 220 -age formula) maximal HR for this sample. Average RPE across the exercise sessions was 13.36 ( $\mathrm{SD}=1.3$ ). 


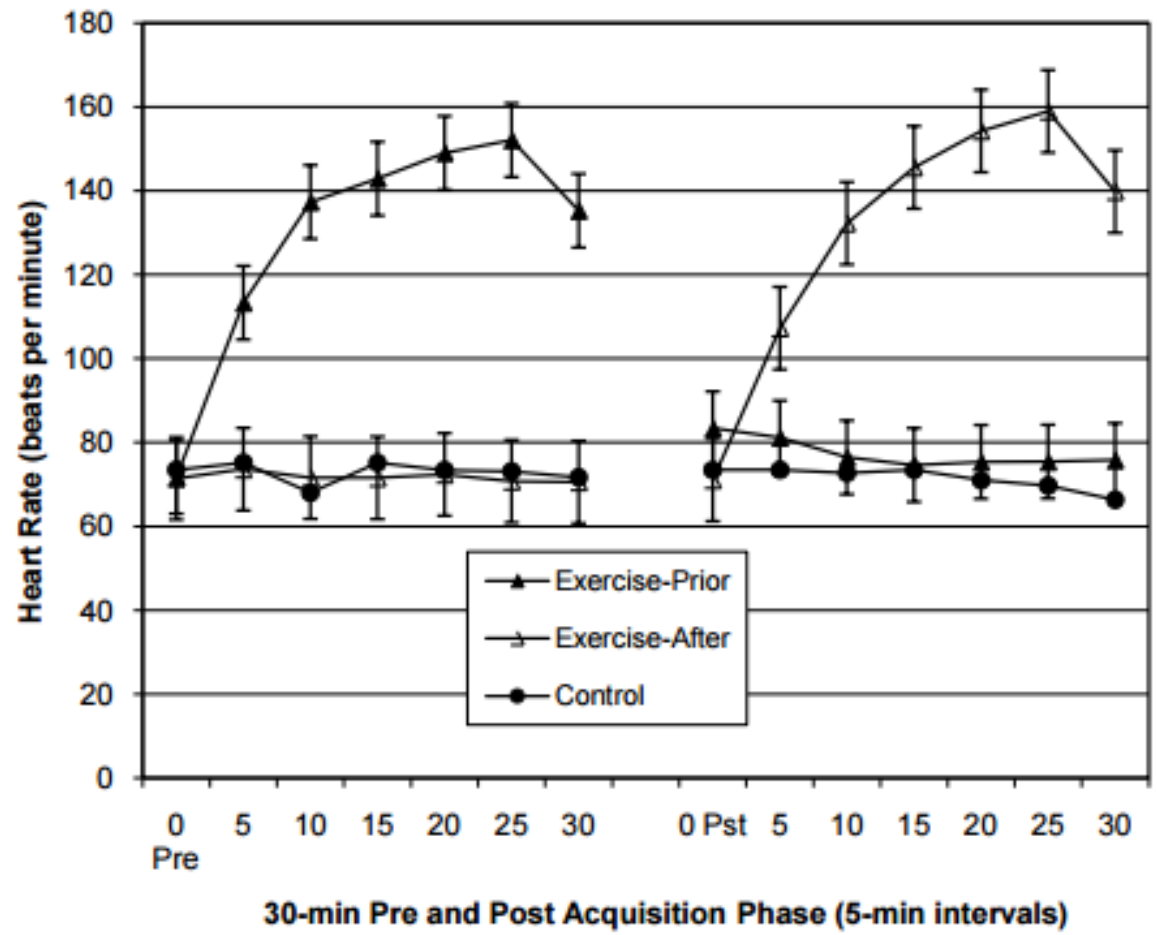

Figure 2. Mean heart rate at 5-min intervals; bars represent standard error.

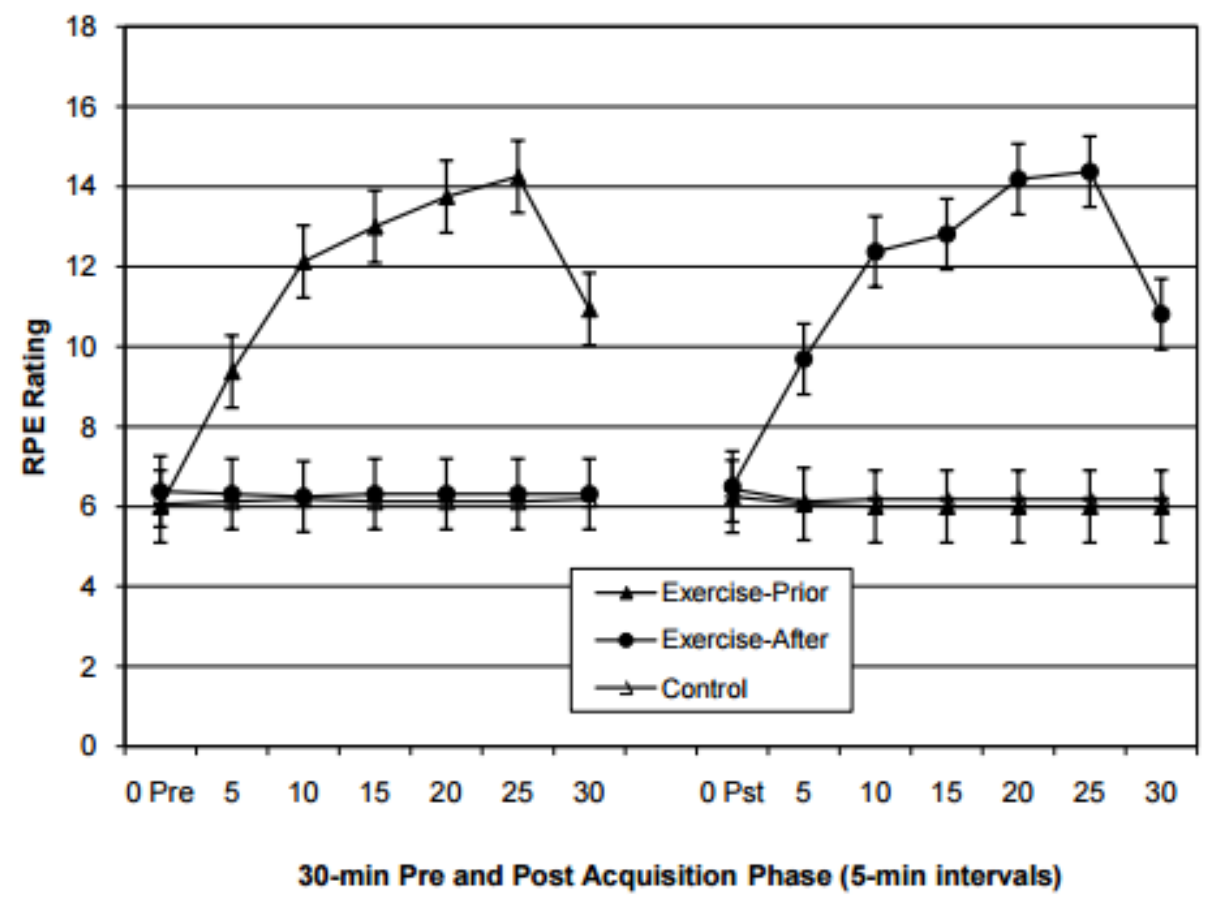

Figure 3. Mean rating of perceived exertion (RPE) at 5-min intervals; bars represent standard error. 


\section{Long-Term Recall Performance}

A one-way ANOVA revealed significant between groups differences in mean delayed recall score, $\mathrm{F}(2,45)=4.37, \mathrm{p}<.05, \eta \mathrm{p}^{2}=0.16$. The mean number of paragraph elements correctly recalled after the 35-min delay was greatest for the exercise-prior group $(\mathrm{M}=15.50, \mathrm{SD}$ $=4.13)$, followed by the exercise-after group $(\mathrm{M}=12.19, \mathrm{SD}=4.68)$ and then the control group $(\mathrm{M}=11.13, \mathrm{SD}=4.27)$. Post hoc testing using Tukey's HSD revealed that only the exercise-prior group's long-term recall performance was significantly better than the control group, $\mathrm{p}<.05$. Performance differences between exercise-prior and exercise-after groups did not reach statistical significance $(\mathrm{p}=.09)$.

Effect sizes for the differences between groups revealed large positive effects (Cohen, 1992) on performance for the exercise-prior group over the control group $(\mathrm{d}=1.04)$ and for the exercise-prior group over the exercise-after group $(\mathrm{d}=0.75)$. Comparisons also revealed a small positive effect of the exercise-after group performance over the control group $(d=0.24)$.

\section{Discussion}

The primary purpose of this study was to determine whether acute aerobic exercise of moderate intensity and duration had an effect on a long-term memory task. A second purpose was to determine if that effect was influenced by the timing of exercise participation relative to the consolidation period. Importantly, mean HR and RPE patterns showed participants kept their RPEs within the desired range, the exercise bout was at a moderate intensity level, and the exercise groups completed bouts comparable in intensity.

The hypothesis that both groups completing a single bout of aerobic exercise would perform better on the long-term memory task was partially supported. Both exercise groups did recall more items than the control group, although post hoc testing revealed that only the exerciseprior group recalled significantly more story items than the control group. An examination of effect sizes indicated that, compared to the control group, exercising prior to the consolidation period resulted in a large effect, while exercising during the consolidation period resulted in a small effect (Cohen, 1992). Although there was a large effect for recall for the exercise-prior group over the exercise-after group, the differences did not reach statistical significance.

However, a power analysis revealed that an increase from 16 to 27 participants per group would likely yield significant differences between these two groups. These results contradict some of the earlier literature suggesting that acute exercise has no effect on long-term recall (Sjoberg, 1980; Tomporowski, 2003; Tomporowski et al., 1987) but are in line with more recent empirical research (Coles \& Tomporowski, 2008; Potter \& Keeling, 2005). One possible explanation for the discrepant results might be the differences in the exercise protocol duration and intensity. Many of the early empirical studies, which failed to support an effect of exercise on long-term recall included elements that might have undermined any positive influences of physical activity on memory. In contrast, more recent studies have tended to support a beneficial effect of exercise on long-term recall, and these studies are characterized by exercise of moderate intensity and duration.

Arousal has long been posited as a possible mechanism to increase exercise-induced cognitive performance, most notably described by the Inverted-U hypothesis (Davey, 1973). Empirical research has not consistently supported this notion (Chang \& Etnier, 2009; Côté, Salmela, \& Papathanasopoulu, 1992; McMorris \& Graydon, 2000; Sjoberg, 1980; Sjoberg, Ohlsson, \& Dornic,1975). An alternative explanation may be that other processes related to 
physiological arousal, such as increases in catecholamines (Zouhal, Jacob, Delamarche, \& GratasDelamarche, 2008) or neurotrophins (Neeper, GomezPinilla, Choi, \& Cotman, 1996; Vaynman \& Gomez-Pinilla, 2005), may drive improvements in cognitive performance. Although neurochemical levels were not directly evaluated in the present study, it has been well established that significant increases can be observed in response to exercise of mild-to-moderate intensity and moderate duration (Hartley et al., 1972; Kjaer, Christensen, Sonne, Richter, \& Galbo, 1985; Zouhal et al., 2008). Thus, when examining the impact of exercise-induced physiological arousal on memory, it appears that activity duration and intensity are important considerations.

\section{Delayed Recall}

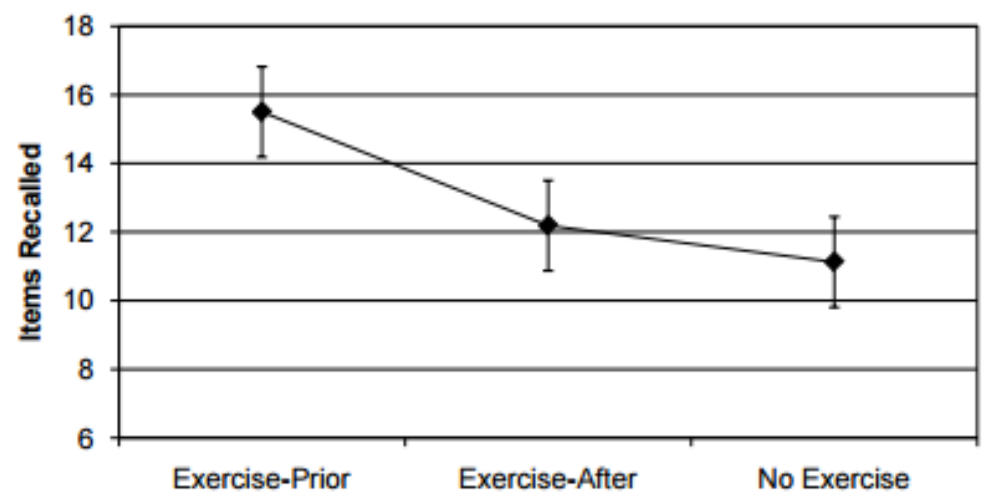

Figure 4. Mean number of paragraph items correctly recalled following a 35-min delay; bars represent standard deviation.

Recall delay may also play an important role in the impact of acute exercise on memory. Findings from a recent study comparing pre- to posttest performance on immediate and delayed recall tasks among participants in exercise and no-exercise conditions (Coles \& Tomporowski, 2008) supported the notion that acute aerobic exercise might benefit certain aspects of long-term, but not short-term, memory. In that study, immediate recall (short-term memory) was assessed via trigram recall (three-letter strings) following delays of 0-18 s. Long-term memory was assessed via recall of word lists following a 12-min delay. Although there was no main effect for condition on immediate recall performance, only participants in the exercise group recalled primacy and recency items (words appearing near the beginning or end of the lists) following a delay. Using longer delays allows for consolidation of acquired information to take place. Results of the present study support this conclusion, in that there were significant results following a 35-min delay. This is also consistent with the assertions by Revelle and Loftus (1992) concerning the minimum delay typically needed to observe a facilitative effect of increased arousal on memory. Perhaps the most novel contribution of the present study was the systematic examination of exercise timing relative to story exposure and consolidation periods. We expected the group participating in acute exercise prior to exposure and consolidation would perform better than either the control group or exercise-after group. This hypothesis was also partially confirmed. Similar to the arousal literature (Abercrombie, Kalin, Thurow, Rosenkranz, \& Davidson, 2003; Cahill \& Alkire, 2003; Cahill, Gorski, \& Le, 2003; McGaugh, 2000; Revelle \& Loftus, 1992; Rimmele, Domes, Mathiak, \& Hautzinger, 2003; Schwabe, Bohringer, Chatterjee, \& Schachinger, 2008), when arousal was elevated prior to exposure and consolidation, recall performance was best. In fact, we found a large effect size when comparing the exercise prior group's performance 
to the control group, as well as the exercise-after group. Post hoc testing, however, did not reveal a statistically significant difference in recall between exercise groups. Thus, timing of the exercise bout appears to be instrumental in observing the effect of exercise on long-term memory.

There are several possible limitations of this study. One is the unequal number of men and women in the sample. There is evidence of gender differences in recall rates involving verbal memory (Herlitz et al., 1997; Herlitz \& Yonker, 2002; Kimura \& Seal, 2003; Kramer et al., 1988; Maitland et al., 2004; Ragland et al., 2000). To address this possible confound, condition assignment was matched such that each group contained the same number of men (5) and women (11). Therefore, gender could not have systematically influenced the different findings between the treatment conditions. However, the literature on gender differences has reported that women exhibit higher verbal recall rates than men. Therefore, a study with more men may not yield a similar magnitude of effect. A second possible limitation is that we did not assess HR during exposure. Thus, we do not know how quickly participants' HR returned to baseline following exercise. Although HR is not the most likely mechanism, it may indicate other arousal processes affecting results. Therefore, it would be useful for future studies to include an index of arousal level during exposure. Last, no physical fitness test was used to set and monitor exercise intensity. Rather, we asked participants to exercise at an intensity that maintained an RPE range of 13-15, and we used HR as a check of exercise intensity. Indeed, some may view another limitation as our failure to use a maximal fitness test to set exercise intensity relative to maximum HR or a ventilatory or lactate threshold. Although research has shown that RPE is a reliable tool for monitoring exercise intensity on a cycle ergometer, individual differences influence RPE (Dishman, 1994; Eston \& Williams, 1988). Thus, future research should include more objective and well validated methods of regulating exercise intensity to advance more specific conclusions regarding the relationship between exercise intensity and cognitive outcomes.

In summary, participation in an acute bout of moderately intense aerobic exercise prior to exposure and consolidation resulted in improved performance on a delayed recall task when compared to controls. These results suggest that exercise can have a positive impact on long-term memory and that acute exercise exerts its greatest facilitative effect when it occurs prior to exposure and consolidation rather than during consolidation. It also appears that intensity and duration are important characteristics of physical activity. Prior research indicated that intense exhausting activity (Tomporowski et al., 1987), exercise resulting in fatigue and dehydration (Brisswalter et al., 2002; Cian et al., 2000; Cian et al., 2001), and light, brief activity (Libkuman, Nichols-Whitehead, Griffith, \& Thomas, 1999; Varner \& Ellis, 1998) can have a detrimental effect on memory performance. The results of this and other studies (Coles \& Tomporowski, 2008; Pesce et al., 2009; Potter \& Keeling, 2005) using moderate intensity exercise of a moderate duration have shown a facilitative effect on long-term memory. While the term moderate exercise has been applied to a broad range of protocols, the moderate exercise most likely to produce beneficial effects on cognitive performance has been identified as an intensity range of 40-80\% of maximal oxygen uptake (Brisswalter et al., 2002; McMorris \& Graydon, 2000) with durations of 20-60 min (Tomporowski, 2003). We designed the exercise protocol in the current study (intensity= 13-15 RPE, 74\% age-predicted maximal $\mathrm{HR}$, duration= $30 \mathrm{~min}$ ) to be consistent with the ranges of moderate exercise identified in the acute exercise reviews. Further, the results from both the current study and previous research suggest the timing of an exercise bout relative to exposure is an important consideration when evaluating the impact of exercise on long-term memory. 


\section{References}

Abercrombie, H. C., Kalin, N. H., Thurow, M. E., Rosenkranz, M. A., \& Davidson, R. J. (2003). Cortisol variation in humans affects memory for emotionally-laden and neutral information. Behavioral Neuroscience, 117, 505-516.

Ahmadiasl, N., Alaei, H., \& Hänninen, O. (2003). Effect of exercise on learning, memory, and levels of epinephrine in rats' hippocampus. Journal of Sports Science and Medicine, 2, $106-109$.

Ainsworth, B. E. (2002). The compendium of physical activities tracking guide [code book]. Retrieved from http://prevention.sph.sc.edu/tools/docs/documents_compendium.pdf

Aks, D. J. (1998). Influence of exercise on visual search: Implications for mediating cognitive mechanisms. Perceptual and Motor Skills, 87, 771-783.

Arcelin, R., \& Brisswalter, J. (1999). Performance stability in simultaneous tasks of pedaling and reaction time. Perceptual and Motor Skills, 88, 1193-1199.

Arcelin, R., Brisswalter, J., \& Delignieres, D. (1997). Effect of physical exercise duration on decisional performance. Journal of Human Movement Studies, 32, 123-140.

Arcelin, R., Delignieres, D., \& Brisswalter, J. (1998). Selective effects of physical exercise on choice reaction processes. Perceptual and Motor Skills, 87, 175-185.

Audiffren, M., Tomporowski, P. D., \& Zagrodnik, J. (2009). Acute aerobic exercise and information processing: Modulation of executive control in a random number generation task. Acta Psychologica, 132, 85-95.

Benson, V., \& Marano, M. A. (1998). Current estimates from the National Health Interview Survey, 1995. Vital and Health Statistics, Series 10, 199, 1-428.

Borg, G. V. (1998). Borg's perceived exertion and pain scales. Champaign, IL: Human Kinetics.

Brisswalter, J., Arcelin, R., Audiffren, M., \& Delignieres, D. (1997). Influence of physical exercise on simple reaction time: Effect of physical fitness. Perceptual and Motor Skills, 85, 1019-1027.

Brisswalter, J., Collardeau, M., \& René, A. (2002). Effects of acute physical exercise characteristics on cognitive performance. Sports Medicine, 32, 555-566.

Brisswalter, J., Durand, M., Delignieres, D., \& Legros, P. (1995). Optimal and non-optimal demand in a dual task of pedaling and simple reaction time: Effects on energy expenditure and cognitive performance. Journal of Human Movement Studies, 29, 15-34.

Cahill, L., \& Alkire, M. T. (2003). Epinephrine enhancement of human memory consolidation: Interaction with arousal at encoding. Neurobiology of Learning and Memory, 79, 194198.

Cahill, L., Gorski, L., \& Le, K. (2003). Enhanced human memory consolidation with postlearning stress: Interaction with degree of arousal at encoding. Learning and Memory, 10, 270-274.

Chang, Y.-K., \& Etnier, J. L. (2009). Exploring the dose-response relationship between resistance exercise intensity and cognitive function. Journal of Sport and Exercise Psychology, 31, 640-656.

Cian, C., Barraud, P. A., Melin, B., \& Raphel, C. (2001). Effects of fluid ingestion on cognitive function after heat stress or exercise-induced dehydration. International Journal of Psychophysiology, 4, 243-251. 
Cian, C., Koulmann, N., Barraud, P. A., Raphel, C., Jimenez, C., \& Melin, B. (2000). Influence of variations in body hydration on cognitive function: Effect of hyperhydration, heat stress, and exercise-induced dehydration. Journal of Psychophysiology, 14, 29-36.

Cohen, J. (1992). A power primer. Psychological Bulletin, 112, 155-159.

Coles, K., \& Tomporowski, P. D. (2008). Effects of acute exercise on executive processing, shortterm and long-term memory. Journal of Sport Sciences, 26, 333-344.

Collardeau, M., Brisswalter, J., \& Audiffren, M. (2001). Effects of a prolonged run on simple reaction time of well trained runners. Perceptual and Motor Skills, 93, 679-689.

Côté, J., Salmela, J., \& Papathanasopoulu, K. P. (1992). Effects of progressive exercise on attentional focus. Perceptual and Motor Skills, 75, 351-354.

Crook, T., Gilbert, J. G., \& Ferris, S. (1980). Operationalizing memory impairment for elderly persons: The Guild Memory Test. Psychological Reports, 47, 1315-1318.

Davey, C. P. (1973). Physical exertion and mental performance. Ergonomics, 16, 595-599.

Davranche, K., Burle, B., Audiffren, M., \& Hasbroucq, T. (2005). Information processing during physical exercise: A chronometric and electromyographic study. Experimental Brain Research, 165, 532-540.

Davranche, K., Burle, B., Audiffren, M., \& Hasbroucq, T. (2006). Physical exercise facilitates motor processes in simple reaction time performance: An electromyographic analysis. Neuroscience Letters, 396, 54-56.

Davranche, K., \& McMorris, T. (2009). Specific effects of acute moderate exercise on cognitive control. Brain and Cognition, 69, 565-570.

Dishman, R. K. (1994). Prescribing exercise intensity for healthy adults using perceived exertion. Medicine \& Science in Sports \& Exercise, 26, 1087-1094.

Dunbar, C. C., Robertson, R. J., Baun, R., Blandin, M. F., Metz, K., Burdett, R., \& Goss, F. L. (1992). The validity of regulating exercise intensity by ratings of perceived exertion. Medicine \& Science in Sports \& Exercise, 24, 94-99.

Emery, C. F., Honn, V. J., Frid, D. J., Lebowitz, K. R., \& Diaz, P. T. (2001). Acute effects of exercise on cognition in patients with chronic obstructive pulmonary disease. American Journal of Respiratory and Critical Care Medicine, 164, 1624-1627.

Eston, R. G., \& Williams, J. G. (1988). Reliability of ratings of perceived effort regulation of exercise intensity. British Journal of Sports Medicine, 22, 153-155.

Etnier, J. L., Salazar, W., Landers, D. M., Petruzzello, S. J., Hahn, M., \& Priscilla, N. (1997). The influence of physical fitness and exercise upon cognitive functioning: A meta-analysis. Journal of Sport and Exercise Psychology, 19, 249-277.

Gilbert, J. G., \& Levee, R. F. (1971). Patterns of declining memory. Journal of Gerontology, 16, $70-75$.

Gilbert, J. G., Levee, R. F., \& Catalano, F. L. (1968). A preliminary report on a new memory scale. Perceptual and Motor Skills, 27, 277-278.

Gilbert, J. G., Levee, R. S., \& Catalano, F. L. (1973). Guild memory test manual. Newark, NJ: Unico National Mental Health Research Center.

Hartley, L. H., Mason, J. W., Hogan, R. P., Jones, L. G., Kotchen, T. A., Mougey, E. H.,...Ricketts, P. T. (1972). Multiple hormonal responses to graded exercise in relation to physical training. Journal of Applied Physiology, 33, 602-606.

Herlitz, A., Nilsson, L.-G., \& Backman, L. (1997). Gender differences in episodic memory. Memory \& Cognition, 25, 801-811. 
Herlitz, A., \& Yonker, J. E. (2002). Sex differences in episodic memory: The influence of intelligence. Journal of Clinical and Experimental Neuropsychology, 24, 107-114.

Hillman, C. H., Snook, E. M., \& Jerome, G. J. (2003). Acute cardiovascular exercise and executive control function. International Journal of Psychophysiology, 48, 307-314.

Hogervorst, E., Riedel, W., Jeukendrup, A., \& Jolles, J. (1996). Cognitive performance after strenuous physical exercise. Perceptual and Motor Skills, 83, 479-488.

Hogervorst, E., Riedel, W. J., Kovacs, E., Brouns, F., \& Jolles, J. (1999). Caffeine improves cognitive performance after strenuous physical exercise. International Journal of Sports Medicine, 20, 354-361.

Kamijo, K., Nishihira, Y., Hatta, A., Kaneda, T., Wasaka, T., Kida, T., \& Kuroiwa, K. (2004). Differential influences of exercise intensity on information processing in the central nervous system. European Journal of Applied Physiology, 92, 305-311.

Kamijo, K., Nishihira, Y., Higashiura, T., \& Kuroiwa, K. (2007). The interactive effect of exercise intensity and task difficulty on human cognitive processing. International Journal of Psychophysiology, 65, 114-121.

Kimura, D., \& Seal, B. N. (2003). Sex differences in recall of real or nonsense words. Psychological Reports, 93, 263-264.

Kjaer, M., Christensen, N. J., Sonne, B., Richter, E. A., \& Galbo, H. (1985). Effect of exercise on epinephrine turnover in trained and untrained male subjects. Journal of Applied Physiology, 59, 1061-1067.

Kramer, J. H., Delis, D. C., \& Daniel, M. (1988). Sex differences in verbal learning. Journal of Clinical Psychology, 44, 907-915.

Kubesch, S., Bretschneider, V., Freudenmann, R., Weidenhammer, N., Lehmann, M., Spitzer, M., $\&$ Gron, G. (2003). Aerobic endurance exercise improves executive functions in depressed patients. Journal of Clinical Psychiatry, 64, 1005-1012.

Lambourne, K., Audiffren, M., \& Tomporowski, P. D. (2010). Effects of acute exercise on sensory and executive processing tasks. Medicine \& Science in Sports \& Exercise, 42, 1396-1402.

Libkuman, T. M., Nichols-Whitehead, P., Griffith, J., \& Thomas, R. (1999). Source of arousal and memory for detail. Memory \& Cognition, 27, 166-190.

Maitland, S. B., Herlitz, A., Nyberg, L., Backman, L., \& Nilsson, L.-G. (2004). Selective sex differences in declarative memory. Memory and Cognition, 32, 1160-1169.

McGaugh, J. L. (1989). Involvement of hormonal and neuromodulatory systems in the regulation of memory storage. Annual Review of Neuroscience, 12, 255-287.

McGaugh, J. L. (2000). Memory: A century of consolidation. Science, 287, 248-251.

McMorris, T., \& Graydon, J. (1996). The effect of exercise on the decision-making performance of experienced and inexperienced soccer players. Research Quarterly for Exercise and Sport, 67, 109-114.

McMorris, T., \& Graydon, J. (2000). The effect of incremental exercise on cognitive performance. International Journal of Sport Psychology, 31, 66-81.

McMorris, T., Myers, S., MacGillivary, W. W., Sexsmith, J. R., Fallowfield, J., Graydon, J., \& Forster, D. (1999). Exercise, plasma catecholamine concentrations and decision-making performance of soccer players on a soccer-specific test. Journal of Sports Sciences, 17, 667-676. 
Meeusen, R., Thorre, K., Chaouloff, F., Sarre, S., De Meirleir, K., Ebinger, G., \& Michotte, Y. (1996). Effects of tryptophan and/or acute running on extracellular 5-HT and 5-HIAA levels in the hippocampus of food-deprived rats. Brain Research, 740, 245-252.

Neeper, S. A., Gomez-Pinilla, F., Choi, J., \& Cotman, C. W. (1996). Physical activity increases mRNA for brain-derived neurotrophic factor and nerve growth factor in rat brain. Brain Research, 726, 49-56.

Netz, Y., Tomer, R., Axelrad, S., Argov, E., \& Inbar, O. (2007). The effect of a single aerobic training session on cognitive flexibility in late middle-aged adults. International Journal of Sports Medicine, 28, 82-87.

Pesce, C., Crova, C., Cereatti, L., Casella, R., \& Bellucci, M. (2009). Physical activity and mental performance in preadolescents: Effects of acute exercise on free-recall memory. Mental Health and Physical Activity, 2(1), 16-22.

Pontifex, M. B., Hillman, C. H., Fernhall, B., Thompson, K. M., \& Valentini, T. A. (2009). The effect of acute aerobic and resistance exercise on working memory. Medicine \& Science in Sports \& Exercise, 41, 927-934.

Potter, D., \& Keeling, D. (2005). Effects of moderate exercise and circadian rhythms on human memory. Journal of Exercise and Sport Psychology, 27, 117-125.

Ragland, J. D., Coleman, A. R., Gur, R. C., Glahn, D. C., \& Gur, R. E. (2000). Sex differences in brain-behavior relationships between verbal episodic memory and resting regional cerebral blood flow. Neuropsychologia, 38, 451-461.

Revelle, W., \& Loftus, D. A. (1992). The implications of arousal effects for the study of affect and memory. In S. A. Christianson (Ed.), Handbook of emotion and memory (pp. 113150). Hillsdale, NJ: Erlbaum.

Rimmele, U., Domes, G., Mathiak, K., \& Hautzinger, M. (2003). Cortisol has different effects on human memory for emotional and neutral stimuli. NeuroReport, 14, 2485-2488.

Schwabe, L., Bohringer, A., Chatterjee, M., \& Schachinger, H. (2008). Effects of pre-learning stress on memory for neutral, positive and negative words: Different roles of cortisol and automatic arousal. Neurobiology of Learning and Memory, 90, 44-53.

Scott, J. P. R., McNaughton, L. R., \& Polman, R. C. J. (2006). Effects of sleep deprivation and exercise on cognitive, motor performance and mood. Physiology and Behavior, 87, 396408.

Sibley, B. A., \& Beilock, S. L. (2007). Exercise and working memory: An individual differences investigation. Journal of Sport and Exercise Psychology, 29, 783-791.

Sjoberg, H. (1980). Physical fitness and mental performance during and after work. Ergonomics, 23, 977-985.

Sjoberg, H., Ohlsson, M., \& Dornic, S. (1975). Physical fitness, work load, and mental performance. Report from the Department of Psychology, University of Stockholm, No. 444.

Soya, H., Nakamura, T., Deocaris, C. C., Kimpara, A., Iimura, M., Fujikawa, T.,... Nishijima, T. (2007). BDNF induction with mild exercise in the rat hippocampus. Biochemical and Biophysical Research Communications, 358, 961-967.

Tomporowski, P. D. (2003). Effects of acute bouts of exercise on cognition. Acta Psychologica, $112,297-324$.

Tomporowski, P. D., Cureton, K., Armstrong, L. E., Kane, G. M., Sparling, P. B., \& MillardStafford, M. (2005). Short-term effects of aerobic exercise on executive processes and emotional reactivity. International Journal of Sport and Exercise. Psychology, 3, 131-146. 
Tomporowski, P. D., \& Ellis, N. R. (1986). Effects of exercise on cognitive processes: A review. Psychological Bulletin, 99, 338-346.

Tomporowski, P. D., Ellis, N. R., \& Stephens, R. (1987). The immediate effects of strenuous exercise on free-recall memory. Ergonomics, 30, 121-129.

Travlos, A. K., \& Marisi, D. Q. (1995). Information processing and concentration as a function of fitness level and exercise-induced activation to exhaustion. Perceptual and Motor Skills, $80,15-26$.

Varner, L. J., \& Ellis, H. C. (1998). Cognitive activity and physiological arousal: Processes that mediate mood-congruent memory. Memory \& Cognition, 26, 939-950.

Vaynman, S., \& Gomez-Pinilla, F. (2005). License to run: Exercise impacts functional plasticity in the intact and injured central nervous system by using neurotrophins. Neurorehabilitation and Neural Repair, 19, 283-295.

Wechsler, D. (1997). Wechsler memory scale (3rd ed.). San Antonio, TX: The Psychological Corporation

Weiss, T. W., Slater, C. H., Green, L. W., Kennedy, V. C., Albright, D. L., \& Wun, C. (1990). The validity of single-item, self-assessment questions as measures of adult physical activity. Journal of Clinical Epidemiology, 43, 1123-1129.

Welsh, R. S., Davis, J. M., Burke, J. R., \& Williams, H. G. (2002). Carbohydrates and physical/mental performance during intermittent exercise to fatigue. Medicine \& Science in Sports \& Exercise, 34, 723-731.

Winter, B., Breitenstein, C., Mooren, F. C., Voelker, K., Fobker, M., Lechtermann, A.,...Knecht, S. (2007). High impact running improves learning. Neurobiology of Learning and Memory, 87, 597-609.

Zouhal, H., Jacob, C., Delamarche, P., \& Gratas-Delamarche, A. (2008). Catecholamines and the effects of exercise, training and gender. Sports Medicine, 38, 401-423.

\section{Authors' Notes}

This research was supported in part by the Susan Stout Graduate Student Research Fellowship. We extend sincere thanks to the donor family and trust of this award, as well as to the delegating committee. We also extend special thanks to Kurt Kornatz and Mike Kanefor their invaluable insight and support on this project. Please address correspondence concerning this article to Jeffrey D. Labban, Department of Kinesiology, University of North Carolina at Greensboro, Greensboro, NC 27402.

E-mail: jdlabban@uncg.edu 